\title{
FERMI: the most powerful computational resource for Italian scientists
}

\author{
F. Falciano, E. Rossi ${ }^{\bowtie}$ \\ Supercomputing Applications and Innovation Department, CINECA, Casalecchio di Reno (BO), Italy
}

By the last quarter of 2012 CINECA, the largest super-computing center in IT and one of the most important worldwide, will provide to all the scientific community one of the world fastest supercomputer, the IBM BlueGene-Q, named FERMI to honor the famous Italian physicist. FERMI with its advanced technology and its 2.1 PFlop peak perform, will allow the Italian scientists for the fastest and most advanced technology available worldwide for scientific calculations. CINECA provides High Performance Computing (HPC) infrastructure for Italian and European researchers and operates in the technological transfer sector through high performance scientific computing, the management and development of networks and web based services, and the development of complex information systems for treating large amounts of data. Moreover CINECA has been appointed by the Italian Ministry of Research and University to represent IT in the European HPC research infrastructure (PRACE). The aim of the HPC Department is to develop and promote technical and scientific services for the Italian and European research community. It constantly invests in Supercomputing technologies, thus being able to provide researchers with some of the hardware resources among the most powerful and energy efficient in Europe. Twice a year CINECA will directly award in excess of 40 million processor core hours, to ensure an adequate supply to scientists and engineers for HPC-related research, and thanks to the new supercomputer the core hours awarded will rise above 1 billion. 